\title{
Surgical Exposure of Bilateral Palatally Impacted Upper Canines and Its Proper Alignment with Fixed Appliance in the Dental Arch - A Case Report
}

\author{
Rizvi HM ${ }^{1}$ BDS, Chawdhury HSR ${ }^{2}$ BDS, Rahman ML ${ }^{3}$ BDS and Hossain MZ ${ }^{4}$ BDS, PhD
}

\begin{abstract}
A case of impacted upper canines of 18 years is presented. Any deviation from the normal sequence of development leads to the impaction of teeth. Although the best treatment alternative for impacted teeth is extraction, the same cannot be directly applied to canines. Since canines present with a high demand on aesthetic and functional requirements, alignment of impacted canines into the arch is the best treatment approach. In the present case, a fixed appliance mechanotherapy was used to bring the impacted canines into occlusion. Total treatment time was about 18 months. Post-treatment records demonstrated good vitality of the impacted tooth and the adjacent teeth.
\end{abstract}

Key Words: Upper canines, palatal impaction, fixed appliance. (Ban J Orthod \& Dentofac Orthop, April 2011; Vol1, No. 2, 13-15)

\section{INTRODUCTION}

Treatment of orthodontic patients entails the management of a constantly changing occlusion from the early mixed dentition to the permanent dentition. In a majority of patients, transition from mixed to permanent dentition occurs uneventfully, without tooth impaction or lack of tooth eruption. However, in a few patients canine impaction may occur as a result of a deviation from the normal sequence of development.

Permanent canines are the foundation of an esthetic smile and functional occlusion. ${ }^{1}$ Any factor that interferes with their normal development and eruption can lead to serious consequences. The maxillary canine is the second most commonly impacted tooth after the mandibular third molars, and the frequency of its impaction is fifty times greater than that of the mandibular canines. $^{2}$

Various inter-arch and intra-arch treatment mechanics have been used to direct the eruption of an impacted tooth.3,4,5,6,7 closed eruption technique is one such treatment modality which requires the application of traction forces to an attachment on the impacted tooth $8,9,10$

\section{DIAGNOSIS}

An 18 year old Bangladeshi male patient presented with impacted upper canines and an over-retained primary upper canines. He had a permanent dentition, with a Class I molar relationship and spacing in the upper anterior segment. The overjet was $6 \mathrm{~mm}$ and the overbite was $4 \mathrm{~mm}$. Cephalometry revealed mild antero-posterior skeletal jaw dysplasia attributed mainly to a mildly prognathic maxilla. Upper incisors are tipped forward resulting in an acute Inter incisal angle. Low Mandibuler plane angle [Figure 3]. No palpable bulges were present on the buccal or palatal side of the impacted canines on intraoral examination. Intraoral periapical radiographs taken with tube shift technique and occlusal films confirmed that the impacted canines were on the palatal side. The crown of the impacted tooth was in close proximity to the root of the permanent lateral incisors [Fig 1]. Lower central incisors were missing and lower canines lingually placed. [Fig 1], [Fig 2].
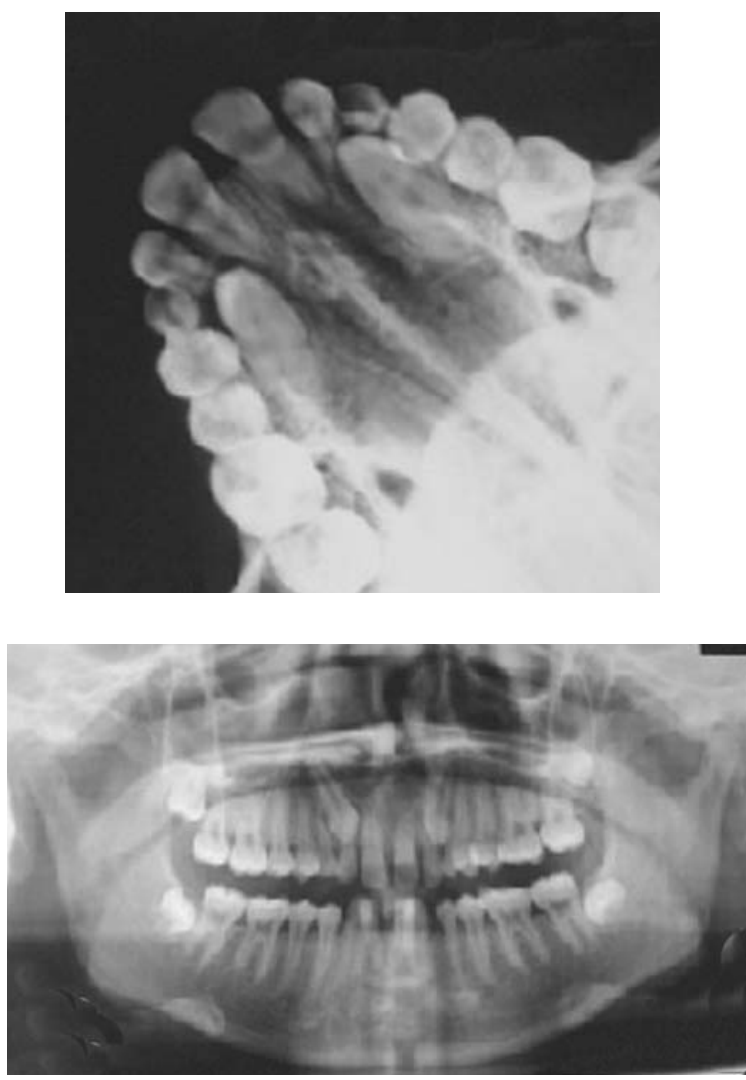

Fig 1. OPG and Occlusal radiograph

${ }^{1-3}$ FCPS-II Trainee, Department of Orthodontics \& Dentofacial Orthopedics, Dhaka Dental College. ${ }^{4}$ Professor \& Head, Department of Orthodontics \& Dentofacial Orthopedics, Dhaka Dental College. 

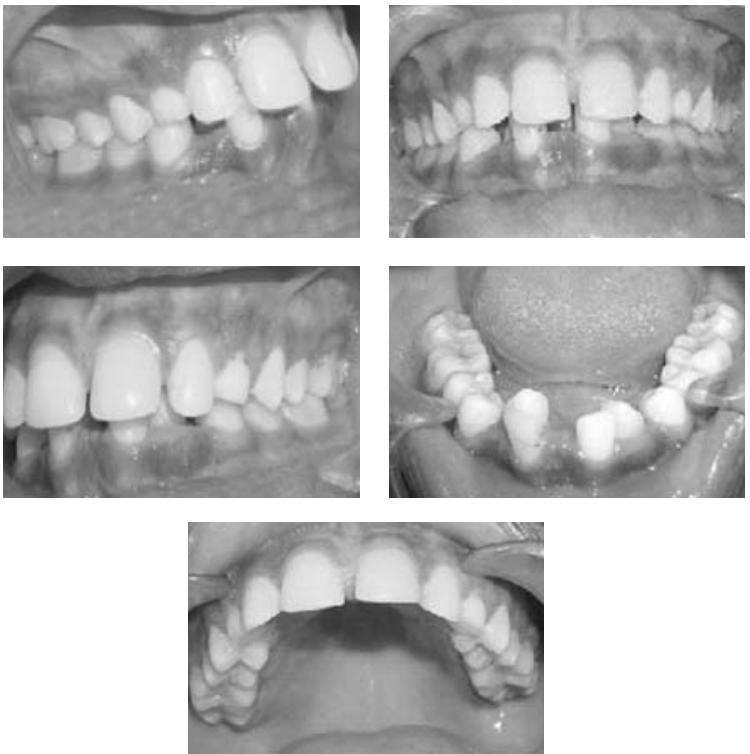

Fig 2. Pre treatment intra oral photographs

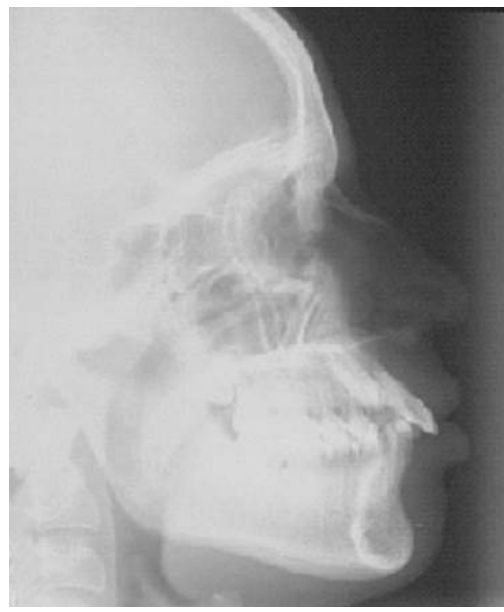

Fig 3. Pre-treatment cephalometric radiographs

\section{TREATMENT PLAN}

1. Extraction of all retained deciduous teeth.

2. Leveling and alignment.

3. Assessment for favorability of eruption and assessment for space adequacy.

4. Surgical exposure of all impacted teeth, removal of overlying bone and reflection of overlying gingival tissue.

5. Traction where necessary and opening up of space for eruption of impacted teeth if necessary.

6. Correction of overjet and overbite.

\section{Surgical Technique}

A palatal flap was raised in the maxilla from left canine region to right canine region under local anesthesia. The crown of the impacted canine was exposed and brackets were bonded to an accessible site on the tooth. A 0.010 " stainless steel ligature wire was passed through the bracket and brought out of the flap.

A tunnel was created through the bone following extraction of the deciduous canines and the flap was then closed with silk sutures, which were to be removed one week later. The fixed appliance was bonded (0.018" x 0.022" standard edgewise) one month before the surgical procedure. The ligature wire was loosely ligated to the arch wire (0.016 inch stainless steel wire) with bilateral cantilever springs. [Fig. 4]

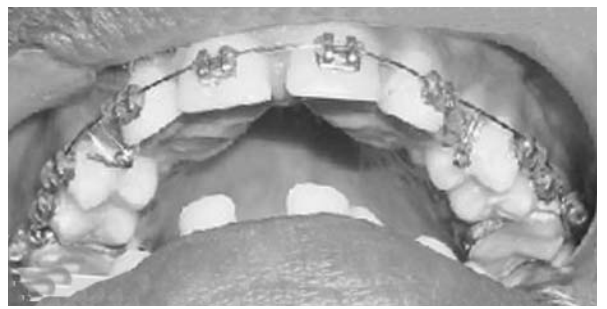

Fig. 4 Impacted canines ligated to the appliance.

\section{CASE PROGRESS}

An incisal eruptive force was applied. Periodic intraoral periapi$\mathrm{cal}$ and occlusal radiographs were taken to assess the angulation and case progress. Direction of pull was changed by changing the position of the cantilver spring on the arch wire.[ Fig. 5]
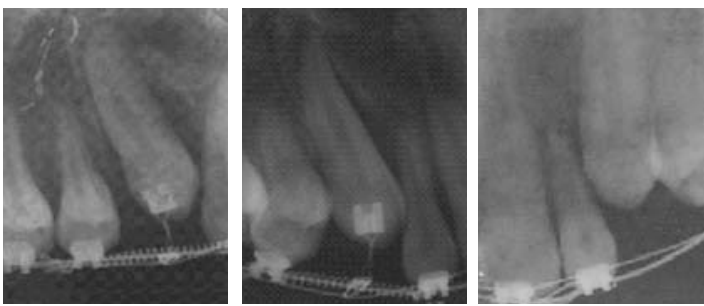

Fig. 5. Periapical $\mathrm{X}$ rays taken to monitor canine angulation/ eruption.
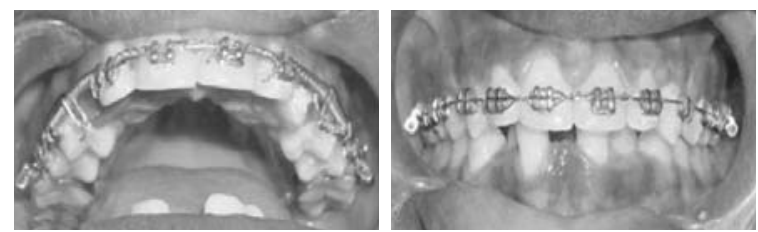

Fig. 6. Continued traction allowed canines to erupt into the arch. 
Bilateral cantilever springs on the piggy back arch wire (0.014inch stainless steel wire) and $\mathrm{Ni}$-Ti open coil spring on the base arch wire (0.018inch stainless steel wire)allowed upper canines to erupt properly into the arch. Once this was accomplished. A 0.014" Nickel Titanium arch wire was then placed for initial leveling and alignment followed by 0.017 " x 0.025 " Nickel Titanium aligning wires. A passive open coil spring was threaded over $0.017 " \mathrm{x} 0.025$ " stainless steel base arch wire for space maintenance in the canine region.

As the impacted canine was reaching its normal level in the arch, a step down bend was given in the stainless steel base arch wire to facilitate further eruption a slight amount of gingival recession was observed with respect to the canines, however this was not found to be clinically significant [Fig. 7]. Leveling of the lower arch used up all spaces.
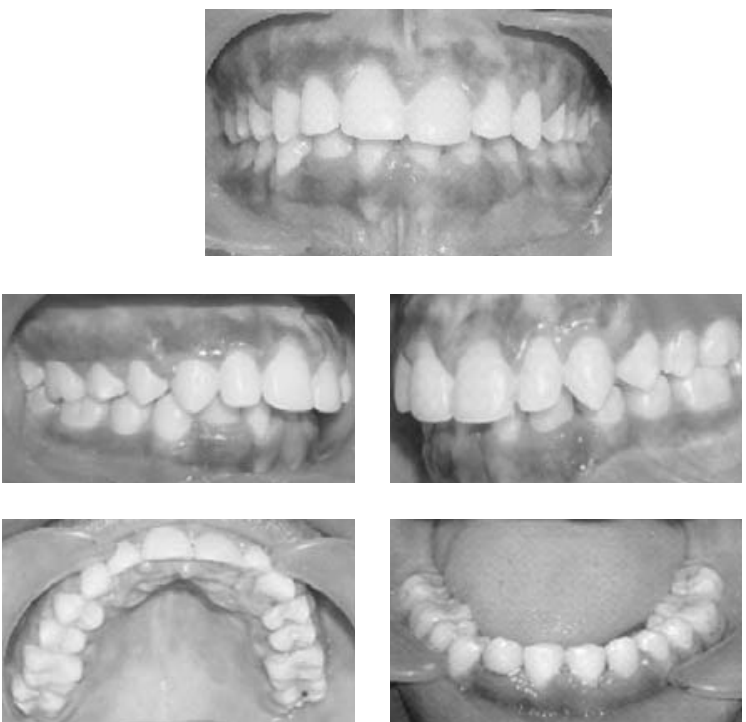

Fig. 7 Post-treatment photographs

\section{DISCUSSION}

The treatment result was satisfactory within the short treatment time. The result was compromised in that sense the increased overjet and overbite were not corrected because the patient did not want to continue any longer for personal reasons he did not want to explain. He requested termination of treatment earlier than the operator intended. A written consent had to be taken that it was the patients will that the operator had to discontinue. However, it was an acceptable occlusion and the patient was happy with his appearance and improved function.

\section{RESULT}

Total treatment time was about 14 months. A minor gingival recession was observed on the canine along with a slight apical root resorption with regard to the adjacent lateral incisor. Both of these findings were, however, insignificant considering the amount of tooth movement required in this case and, most importantly, preserving the vitality of the associated teeth.

\section{CONCLUSION}

The chief advantage of the closed eruption technique over direct bonding is that it maintains the width of the attached gingiva. In addition, the impacted canine was brought into the arch without causing any deleterious effects to the arch form.

\section{ACKNOWLEDGMENTS}

I would like to thank my supervisor Prof. Dr. Md. Zakir Hossain for his help with this case. I would also like to thank senior collogues, technicians and stuff of Department of Orthodontics Dhaka dental College \& Hospital.

\section{REFERENCES}

1. Patrick F. Mcsherry. The ectopic maxillary canine ; A Review. Br. J. Orthod 1998; 25: 209-216

2. Kornhauser S, Abed Y, Harari D, and Becker A. The resolution of palatally impacted canines using palatal- Occlusal force from a buccal auxillary. Am .J. Orthod 1996;110: 528-534.

3. Harry Jacoby. "The Ballista Spring" system for impacted teeth. Am. J. Orthod. 1979; 75: 143-151.

4. Gregory Oppenhuizen. An extrusion spring for palatally impacted cuspids. J. Clin. Orthod 2003; 37: 434-436.

5. S. Jay. Bowman, Aldo Carano. The Kilroy spring for impacted teeth. J. Clin. Orthod. 2003; 37: 683-688.

6. Samuels R. H. A new eruption attachment for impacted teeth. J. Clin. Orthod 2003; 38: 496-500.

7. Samir Bishara. Impacted maxillary canines. Am. J. Orthod 1992; 101: 159-171.

8. Kokich, V.G and Mathews, D.P. Surgical and orthodontic management of impacted teeth. Dent. Clin. N. Am 1993; 37: 181-204.

9. Crescini A, Clauser C, Giorgetti R, Cortellini P and Piniprato, G. P. Tunnel traction of infraosseous impacted canines. A three-year periodontal follow-up. Am. J. Orthod 1994; 105: 61-72.

10. S. Jay Bowman, Aldo Carano. The Monkey Hook: An Auxillary for impacted, rotated, and displaced teeth. J. Clin. Orthod 2002; 36: 375-378

\section{Correspondence}

\section{Dr. Hasan Md. Rizvi, BDS}

FCPS Trainee

Dept. of Orthodontics and Dentofacial Orthopedics

Dhaka Dental College and Hospital

Mirpur 14, Dhaka 1206

Mobile : +8801710918222, E-mail : hasanriz2008@yahoo.com 